\title{
pesquisa
}

TAKAHASHI HW; HIDALGO PC; FADELLI L; CUNHA MET. 2007. Composição e manejo da solução nutritiva visando a diminuição do teor de nitrato nas folhas de alface hidropônica. Horticultura Brasileira 25: 006-009.

\section{Composição e manejo da solução nutritiva visando a diminuição do teor de nitrato nas folhas de alface hidropônica}

\author{
Hideaki W Takahashi ${ }^{1}$; Paulo C Hidalgo ${ }^{2}$; Laércio Fadelli ${ }^{1}$; Magda E T da Cunha ${ }^{1}$ \\ ${ }^{1}$ UEL, Depto. Agronomia, C. Postal 6001, 86051-990 Londrina-PR; EMATER, C. Postal 386, 86300-000 Cornélio Procópio-PR; E- \\ mail:hwilson@uel.br.
}

\begin{abstract}
RESUMO
As hortaliças folhosas têm elevada capacidade de acumular nitrato nas folhas e pecíolos, mas o consumo excessivo de nitrato pode ser prejudicial à saúde humana. Determinou-se a melhor combinação de doses e fontes de $\mathrm{N}$ e época de fornecimento na solução nutritiva para obter diminuição do teor de nitrato em folhas de alface, cv. Vera. Os tratamentos foram (T1) $210 \mathrm{mg} \mathrm{L}^{-1}$ de $\mathrm{N}$ como nitrato $\left(\mathrm{N}^{-\mathrm{NO}_{3}^{-}}\right.$) do transplante à colheita; (T2) $189 \mathrm{mg} \mathrm{L}^{-1}(90 \%)$ de $\mathrm{N}-$ $\mathrm{NO}_{3}^{-}$associado com $21 \mathrm{mg} \mathrm{L}^{-1}(10 \%)$ de $\mathrm{N}$ como amônio $\left(\mathrm{N}^{-\mathrm{NH}_{4}}{ }^{+}\right.$); (T3) $210 \mathrm{mg} \mathrm{L}^{-1}$ de $\mathrm{N}_{-\mathrm{NO}_{3}^{-}}^{-}$até 24 dias após transplante e substituição por $189 \mathrm{mg} \mathrm{L}^{-1}$ de $\mathrm{N}^{-} \mathrm{NO}_{3}^{-}$e $21 \mathrm{mg} \mathrm{L}^{-1}$ de $\mathrm{N}^{-\mathrm{NH}_{4}}{ }_{4}^{-}$até o final do ciclo; (T4) $210 \mathrm{mg} \mathrm{L}^{-1}$ de $\mathrm{N}_{-} \mathrm{NO}_{3}^{-}$até o $24^{\circ}$ dia e redução para105 mg $\mathrm{L}^{-1}$ de $\mathrm{N}_{-} \mathrm{NO}_{3}^{-}$no final do ciclo e (T5) $210 \mathrm{mg} \mathrm{L}^{-1}$ de $\mathrm{N}_{-} \mathrm{NO}_{3}^{-}$até o $24^{\circ}$ dia do transplante e redução para $52,5 \mathrm{mg} \mathrm{L}^{-1}$ de $\mathrm{N}^{-\mathrm{NO}_{3}}$ - no final do ciclo. Os melhores resultados foram obtidos com os tratamentos 2 e 3, obtendo teores de nitrato na parte aérea de 1.756 a $1.920 \mathrm{mg}$ $\mathrm{kg}^{-1}$ na matéria fresca e produtividade equivalente ao tratamento 1 . A redução de nitrato em solução nutritiva no final do ciclo não reduziu o teor de nitrato em folhas.
\end{abstract}

Palavras-chave: Lactuca sativa, cultivo hidropônico, fontes de nitrogênio.

\begin{abstract}
Nutrient solution control in order to decrease nitrate content in leaves of hydroponic lettuce

The edible vegetables have a high capacity to accumulate nitrate in the leaves and stem. The excessive consumption of nitrate can be harmful to human health. The best combination of doses and sources of $\mathrm{N}$ and supply time were determined in the nutritious solution to reduce the nitrate concentration in $\mathrm{cv}$. Vera leaves of lettuce. The treatments were (T1) $210 \mathrm{mg} \mathrm{L}^{-1}$ of $\mathrm{N}$ as nitrate $\left(\mathrm{N}^{-\mathrm{NO}_{3}}{ }^{-}\right)$from transplantation to harvest; (T2) $189 \mathrm{mg} \mathrm{L}^{-1}(90 \%)$ of $\mathrm{N}$ as nitrate (N$\mathrm{NO}_{3}^{-}$) associated with $21 \mathrm{mg} \mathrm{L}^{-1}(10 \%)$ of $\mathrm{N}$ as ammonium $\left(\mathrm{N}^{-\mathrm{NH}_{4}^{+}}\right)$; (T3) $210 \mathrm{mg} \mathrm{L}^{-1}$ of nitrate until the $24^{\text {th }}$ day and substitution for 189 mg L ${ }^{-1}$ of nitrate and $21 \mathrm{mg} \mathrm{L}^{-1} \mathrm{~N}_{-\mathrm{NH}_{4}^{+}}^{+}$until the end of the cycle; (T4) $210 \mathrm{mg} \mathrm{L}^{-1}$ of $\mathrm{N}^{-\mathrm{NO}_{3}^{-}}$until the $24^{\text {th }}$ day of the transplant and reduction to $105 \mathrm{mg} \mathrm{L}^{-1}$ of $\mathrm{N}^{-\mathrm{NO}_{3}-}$ until the end of the cycle; (T5) $210 \mathrm{mg} \mathrm{L}^{-1}$ of $\mathrm{N}^{-\mathrm{NO}_{3}-}$ until the $24^{\text {th }}$ day of the transplant and reduction to $52,5 \mathrm{mg} \mathrm{L}^{-1}$ of $\mathrm{N}^{-\mathrm{NO}_{3}}$ until the end of the cycle. The best results were obtained with the treatments T2 and T3, with levels of nitrate in the edible part varying from 1,756 to $1,920 \mathrm{mg} \mathrm{kg}^{-1}$ in the fresh matter and equivalent yield to the treatment 1 . The reduction of nitrate in nutritious solution in the end of the cycle did not reduce the quantity of nitrate in leaves.
\end{abstract}

Keywords: Lactuca sativa, hydroponic cultivation, nitrogen sources.

(Recebido para publicação em 20 de julho de 2005; aceito em 27 de janeiro de 2007)

$\mathrm{O}$ interesse pelo cultivo em sistema hidropônico vem crescendo nos últimos anos no Brasil. A hidroponia apresenta algumas vantagens como economia de água, automação, produção durante o ano todo, controle da nutrição das plantas e qualidade do produto colhido (Furlani et al., 1999). A alface produzida nesse sistema tem obtido remuneração de 35 a $50 \%$ superior à cultivada no sistema convencional, mesmo em mercados tradicionais como as CEASAS (Junqueira, 1999).

O nitrogênio tem importante função na planta como componente de aminoácidos e proteínas; é absorvido principalmente como nitrato e em menor proporção como amônio, tendo o primeiro também a função de regulador osmótico no vacúolo (Blom-Zandstra, 1989). Períodos de alta luminosidade, como na primavera e no verão, induzem maior atividade da enzima redutase do nitrato, consequentemente reduzindo a quantidade de nitrato pela incorporação em aminoácidos e proteínas. No inverno, se houver alta disponibilidade de $\mathrm{N}$ associada a pouca luminosidade com baixa fotossíntese, o nitrato se acumula no vacúolo da célula e pode atingir níveis elevados (Blom-Zandstra \& Lampe, 1985).

A produção de hortaliças folhosas em hidroponia exige alguns cuidados com a nutrição, podendo ocorrer maior acúmulo de nitrato nas plantas do que no processo convencional de cultivo (Bennini et al., 2002). As hortaliças têm diferentes capacidades para acumular nitrato, sendo espinafre, alface, rúcula, almeirão, nabo, rabanete e beterraba as que têm maiores possibilidades de apresentarem teores elevados em algumas condições de cultivo; folhas e pecíolos têm maior capacidade de acumular nitrato que inflorescências e frutos (Lorenz, 1978).

O nitrato consumido em excesso pode ser prejudicial à saúde, podendo levar à formação de nitrito e causar inibição do transporte de oxigênio no sangue. Outros compostos que podem ser formados são as aminas secundárias como nitrosaminas, Nnitrosodimethilamina que são potencialmente cancerígenas (Fine et al., 1977; Craddock, 1983). 
A maior quantidade de nitrato consumido pela população provém do consumo de hortaliças, representando 72 a 94\% do total ingerido (Sheng Minghzu, 1982). Dados da organização mundial de saúde preconizam que a ingestão diária aceitável de nitrato é de $3,65 \mathrm{mg} \mathrm{kg}^{-1}$ de massa corpórea (Escoín-Peña et al.,1998). A legislação européia define limites para o teor de nitrato na matéria fresca dos vegetais a serem consumidos pela população, sendo $4500 \mathrm{mg} \mathrm{kg}^{-1}$ para inverno e $3000 \mathrm{mg} \mathrm{kg}^{-1}$ para verão os limites para nitrato em cultivos sob ambiente protegido, e $2500 \mathrm{mg} \mathrm{kg}^{-1} \mathrm{o}$ limite de nitrato para o cultivo em campo aberto (Anon, 1997). A legislação brasileira não define teores de nitrato em vegetais consumidos pela população, e tampouco seus teores são monitorados nos vegetais comercializados, apesar da importância desse no sistema de produção.

É possível reduzir o acúmulo de nitrato em partes comestíveis de hortaliças com estratégias como aumentar a intensidade luminosa, colher durante ou logo após um período de alta luminosidade, atrasar a época de colheita, utilizar cultivares com menor capacidade para acumular nitrato, reduzir as doses de nitrato aplicadas durante o ciclo ou no final do ciclo, colocar parte da adubação como amônio e aplicar cloro (Van der Boon et al., 1990; McCall \& Willumsen, 1998; Fernandes et al., 2002).

O objetivo deste trabalho foi avaliar soluções nutritivas distintas quanto à concentração de $\mathrm{N}$ e relação nitratoamônio para diminuir o teor de nitrato em folhas de alface, cv. Vera.

\section{MATERIAL E MÉTODOS}

O experimento foi instalado em 17/ 01/04, em área experimental da UEL, em hidroponia, em casa de vegetação com teto em arco de $6 \mathrm{~m}$ de largura e 15 $\mathrm{m}$ de comprimento, coberta com polietileno de baixa densidade de 100 micrômetros de espessura. Foi empregado o sistema de hidroponia denominado fluxo laminar de nutrientes (NFT), com recirculação de nutrientes, composto por uma bancada de 5 tubos de PVC para hidroponia, com espaçamento de $25 \mathrm{~cm}$.
Para cada tratamento foi utilizado um conjunto composto por um reservatório, um conjunto moto bomba de $1 / 4 \mathrm{CV}$ (184 W) e tubulação de sucção, recalque, retorno e cultivo. Os reservatórios de cimento amianto, impermeabilizados, com capacidade de $50 \mathrm{~L}$ de capacidade e $40 \mathrm{~L}$ úteis foram parcialmente enterrados em areia. $\mathrm{O}$ recalque subterrâneo em tubos de PVC com retorno de parte da vazão ao reservatório foi por tubos perfurados para melhorar a aeração e diminuir a temperatura da solução.

A circulação da solução nutritiva foi processada de forma independente em cada tratamento, sendo os cinco conjuntos conectados e sincronizados por um temporizador funcionando de forma contínua das 10 às 17 horas e de forma intermitente no restante do período com 10 minutos de pausa e 10 minutos de circulação da solução.

A alface crespa cultivar Vera foi semeada em 23/12/03 em bandejas de isopor com 288 células contendo substrato comercial Plantmax ${ }^{\circledR}$. As mudas foram transplantadas em 17/01/04, aos 20 dias da emergência com 2 a 3 pares de folhas para solução hidropônica com produtos "puro para análise", com as seguintes concentrações em $\mathrm{mg} \mathrm{L}^{-1}$ : N-210; P-31; K-191; Ca-200; Mg-48; S69,$4 ; \mathrm{Fe}-2 ; \mathrm{Zn}-0,04 ; \mathrm{Mn}-0,5 ; \mathrm{B}-0,5 ; \mathrm{Cu}-$ 0,015 e Mo- 0,09 .

$\mathrm{O}$ delineamento experimental utilizado foi de blocos ao acaso, com cinco tratamentos e cinco repetições, seis plantas por parcela, sendo as quatro plantas centrais utilizadas para avaliação das características.

Os tratamentos avaliados foram (T1) solução nutritiva (SN) com $210 \mathrm{mg} \mathrm{L}^{-1}$ de N como nitrato em todo ciclo; (T2) $\mathrm{SN}$ com $189 \mathrm{mg} \mathrm{L}^{-1}$ de $\mathrm{N}$ na forma de $\mathrm{NO}_{3}^{-}$e $21 \mathrm{mg} \mathrm{L}^{-1}$ de $\mathrm{N}$ na forma de amônio em todo ciclo; (T3) SN com 210 $\mathrm{mg} \mathrm{L}^{-1}$ de $\mathrm{N}$ na forma de $\mathrm{NO}_{3}^{-}$até o $24^{\circ}$ dia, substituída por $\mathrm{SN}$ com $189 \mathrm{mg} \mathrm{L}^{-1}$ de $\mathrm{N}_{-} \mathrm{NO}_{3}{ }^{-}$e $21 \mathrm{mg} \mathrm{L}^{-1}$ de $\mathrm{N}$ como $\mathrm{NH}_{4}^{+}$ até o final do ciclo.; (T4) SN com 210 $\mathrm{mg} \mathrm{L}^{-1}$ de $\mathrm{N}_{-} \mathrm{NO}_{3}^{-}$até o $24^{\circ}$ dia substituída por SN com $105 \mathrm{mg} \mathrm{L}^{-1}$ de $\mathrm{N}$ na forma de $\mathrm{NO}_{3}^{-}$no restante do ciclo; (T5) $\mathrm{SN}$ com $210 \mathrm{mg} \mathrm{L}^{-1}$ de $\mathrm{N}$ na forma de $\mathrm{NO}_{3}{ }^{-}$até o $24^{\circ}$ dia substituída por 52,5 $\mathrm{mg} \mathrm{L}^{-1}$ de $\mathrm{N}$ na forma de $\mathrm{NO}_{3}^{-}$no restante do ciclo.
Foram monitoradas as temperaturas da solução nutritiva às 8 horas e às 14 horas em todos os tratamentos e a temperatura do ar no interior da estufa. Os níveis de solução nos reservatórios foram completados para $40 \mathrm{~L}$, duas vezes ao dia. Em seguida o $\mathrm{pH}$ era corrigido para valores de 6,2 $\pm 0,2$, com ácido clorídrico ou hidróxido de sódio. A condutividade elétrica (CE) da solução foi monitorada uma vez ao dia e corrigida em todos os tratamentos sempre que houvesse depleção de $20 \%$ no valor da CE inicial, mesmo que ocorresse em apenas um dos tratamentos.

Após 34 dias do transplante, as plantas foram colhidas e separadas em parte aérea e raiz para a determinação da matéria fresca, lavadas com água corrente e secas em estufa com circulação de ar forçada a $55^{\circ} \mathrm{C}$ até peso constante e determinado o peso da matéria seca. A parte aérea foi moída para a determinação de nitrato, conforme metodologia descrita por Cataldo et al. (1975).

As médias dos tratamentos de matéria fresca e seca da parte aérea e raiz e o teor de nitrato da parte aérea foram comparadas pelo teste de Tukey.

\section{RESULTADOS E DISCUSSÃO}

Os resultados deste experimento, com média geral de 301 g de matéria fresca por planta, são superiores aos obtidos por Bennini et al. (2002) em 32 amostras de alface comercial hidropônica coletadas em Londrina-PR, que foi de 271 gramas. Os tratamentos 2 e 3, contendo amônio em solução, apresentaram os menores valores de matéria seca de raiz e da parte aérea (Tabela 1). A presença de amônio em solução proporciona um maior declínio de $\mathrm{pH}$ em soluções nutritivas, especialmente em altas temperaturas (Kafkafi, 1990) e, no caso dos tratamentos 2 e 3 , influenciou a produção de matéria seca da raiz e parte aérea. Mesmo com duas correções diárias de $\mathrm{pH}$ houve maior diminuição de $\mathrm{pH}$, entre as correções nos tratamentos 2 e 3 em relação aos tratamentos com nitrato em solução.

A matéria fresca da parte aérea, que é a parte comercializável, não apresentou diferenças entre os tratamentos 1,2 , 3 e 5 (Tabela 1). 
Tabela 1. Matéria fresca da parte aérea (Mfpa), matéria seca da parte aérea (Mspa), matéria fresca de raiz (Mfr), matéria seca de raiz (Msr), da alface crespa cv. Vera, cultivada com soluções nutritivas de diferentes composições e manejo (Fresh matter of the aerial part (Mfpa), dry matter of the aerial part (Mspa), fresh matter of roots (Mfr) and dry matter of roots (Msr), of crisp lettuce, cv. Vera, cultivated with nutritive solutions and various compositions). Londrina, UEL, 2004.

\begin{tabular}{|c|c|c|c|c|}
\hline \multirow{2}{*}{ Tratamento } & Mfpa & Mspa & $\mathrm{Mfr}$ & Msr \\
\hline & \multicolumn{4}{|c|}{$\left(\right.$ g planta $\left.^{-1}\right)$} \\
\hline $\begin{array}{l}210 \mathrm{mg} \mathrm{L}^{-1} \text { de } \mathrm{N} \text { na forma de } \mathrm{NO}_{3}^{-} \mathrm{em} \\
\text { todo ciclo }\end{array}$ & $337 a^{*}$ & $337 a^{*}$ & $51,4 \mathrm{a}$ & $2,31 \mathrm{~b}$ \\
\hline $\begin{array}{l}189 \mathrm{mg} \mathrm{L}^{-1} \text { de } \mathrm{N} \text { na forma de } \mathrm{NO}_{3}^{-} \text {e } 21 \\
\mathrm{mg} \mathrm{L}^{-1} \text { de } \mathrm{N} \text { na forma de } \mathrm{NH} 4+\text { em todo } \\
\text { ciclo }\end{array}$ & $291 \mathrm{a} \mathrm{b}$ & $13,85 \quad b$ & $40,1 \quad b$ & $1,79 \mathrm{c}$ \\
\hline $\begin{array}{l}210 \mathrm{mg} \mathrm{L}^{-1} \text { de } \mathrm{N} \text { na forma de } \mathrm{NO}_{3}^{-} \text {até } o \\
24^{\circ} \text { dia, substituída por } 189 \mathrm{mg} \mathrm{L}^{-1} \text { de } \mathrm{N} \\
\text { na forma de } \mathrm{NO}_{3}^{-} \text {e } 21 \mathrm{mg} \mathrm{L}^{-1} \text { de } \mathrm{N} \text { na } \\
\text { forma de } \mathrm{NH}_{4}^{+} \text {do } 24^{\circ} \text { dia até a colheita }\end{array}$ & $289 a b$ & $13,87 \quad b$ & $37,6 \quad b$ & $2,01 \mathrm{~b} \mathrm{c}$ \\
\hline $\begin{array}{l}210 \mathrm{mg} \mathrm{L}^{-1} \text { de } \mathrm{N} \text { na forma de } \mathrm{NO}_{3}^{-} \text {até o } \\
24^{\circ} \text { dia, substituída por } 105 \mathrm{mg} \mathrm{L}^{-1} \text { do } \\
24^{\circ} \text { dia até a colheita }\end{array}$ & $285 b$ & $15,40 \mathrm{a} \mathrm{b}$ & 37,6 b & $2,38 \mathrm{~b}$ \\
\hline $\begin{array}{l}210 \mathrm{mg} \mathrm{L}^{-1} \text { de } \mathrm{N} \text { na forma de } \mathrm{NO}_{3}^{-} \text {até o } \\
24^{\circ} \text { dia, substituída por } 52,5 \mathrm{mg} \mathrm{L}^{-1} \text { do } \\
24^{\circ} \text { dia até a colheita }\end{array}$ & 302 a b & $15,53 \mathrm{ab}$ & 45,2 a & $2,88 \mathrm{a}$ \\
\hline Média & 301 & 15,17 & 42,38 & 2,27 \\
\hline C.V.\% & 8,60 & 8,64 & 11,17 & 10,16 \\
\hline
\end{tabular}

*Médias seguidas da mesma letra na coluna não diferem a $5 \%$ de probabilidade para matéria fresca e $1 \%$ para matéria seca pelo teste de Tukey (means followed by the same letter in the column did not differ from each other by the Tukey test, $\mathrm{P}<0.05$ ).

Tabela 2. Teores de nitrato na matéria fresca de alface crespa, cv. Vera, cultivada com soluções nutritivas de diferentes composições e manejo (Nitrate levels in the fresh matter of crisp lettuce, cv. Vera, cultivated under different compositions and use of nutritive solutions). Londrina, UEL, 2004.

\begin{tabular}{|c|c|}
\hline Tratamento & $\begin{array}{l}\text { Nitrato } \\
\left(\mathrm{mg} \mathrm{kg}^{-1}\right)\end{array}$ \\
\hline $210 \mathrm{mg} \mathrm{L}^{-1}$ de $\mathrm{N}$ na forma de $\mathrm{NO}_{3}^{-}$em todo ciclo & $2407 b^{*}$ \\
\hline $\begin{array}{l}189 \mathrm{mg} \mathrm{L}^{-1} \text { de } \mathrm{N} \text { na forma de } \mathrm{NO}_{3}^{-} \text {e } 21 \mathrm{mg} \mathrm{L}^{-1} \text { de } \mathrm{N} \text { na forma de } \\
\mathrm{NH}_{4}^{+} \text {em todo ciclo }\end{array}$ & $1920 \mathrm{~d}$ \\
\hline $\begin{array}{l}210 \mathrm{mg} \mathrm{L}^{-1} \text { de } \mathrm{N} \text { na forma de } \mathrm{NO}_{3}^{-} \text {até } 24^{\circ} \text { dia substituída por } 189 \\
\mathrm{mg} \mathrm{L}^{-1} \text { de } \mathrm{N} \text { na forma de } \mathrm{NO}_{3}^{-} \text {e } 21 \mathrm{mg} \mathrm{L}^{-1} \text { de } \mathrm{N} \text { na forma de } \mathrm{NH}_{4}^{+} \text {do } \\
24^{\circ} \text { dia até a colheita }\end{array}$ & $1756 \mathrm{~d}$ \\
\hline $\begin{array}{l}210 \mathrm{mg} \mathrm{L}^{-1} \text { de } \mathrm{N} \text { na forma de } \mathrm{NO}_{3}^{-} \text {até } 24^{\circ} \text { dia substituída por } 105 \\
\mathrm{mg} \mathrm{L}^{-1} \text { do } 24^{\circ} \text { dia até a colheita }\end{array}$ & 3024 a \\
\hline $\begin{array}{l}210 \mathrm{mg} \mathrm{L}^{-1} \text { de } \mathrm{N} \text { na forma de NO3- até } 24^{\circ} \text { dia substituída por } 52,5 \\
\mathrm{mg} \mathrm{L}^{-1} \text { do } 24^{\circ} \text { dia até a colheita }\end{array}$ & $2465 b$ \\
\hline Média & 2314 \\
\hline C.V \% & 11,13 \\
\hline
\end{tabular}

*Médias seguidas por mesma letra na coluna não diferem a $1 \%$ de probabilidade pelo teste de Tukey (means followed by the same letter in the column did not differ from each other by the Tukey test, $\mathrm{P}<0.05$ ).

O teor médio de nitrato de $2314 \mathrm{mg}$ $\mathrm{kg}^{-1}$ (Tabela 2) é superior à média geral de $1588 \mathrm{mg} \mathrm{kg}^{-1}$ encontrada por Bennini et al. (2002) em 32 amostras de alface hidropônica comercial, porém abaixo do limite máximo de $2500 \mathrm{mg} \mathrm{kg}^{-1}$ de nitrato em folhas, admitido pela legislação européia, com exceção do tratamento 4, diminuição de sua disponibilidade em solução, porém isto não ocorreu neste experimento. Os tratamentos 4 e 5, com menores concentrações de $\mathrm{N}^{-\mathrm{NO}_{3}}{ }^{-}$em solução nos últimos 10 dias do final do ciclo, não apresentaram redução do teor de nitrato na parte aérea. $\mathrm{O}$ tratamento 4 apresentou o maior teor de nitrato, pois houve concentração do mesmo em função do menor acúmulo de matéria fresca neste tratamento.

Economakis et al. (1997), trabalhando com concentrações de 50, 100 e 150 $\mathrm{mg} \mathrm{L}^{-1}$ de $\mathrm{N}$ como nitrato em solução nutritiva, não obtiveram diferenças de produtividade e teor de nitrato na parte aérea da alface. Reinink \& Eenink (1988) mostraram em experimento com cultivares de alface em soluções contendo 96,161 e $189 \mathrm{mg} \mathrm{L}^{-1}$ de $\mathrm{N}_{-} \mathrm{NO}_{3}^{-}$haver baixa correlação entre teor de nitrato no caule, na raiz e matéria fresca da planta e concluíram que o conteúdo de nitrato é complexo e influenciado por diversos mecanismos fisiológicos.

Schoubeck et al. (1991), reduzindo o nitrato na solução nutritiva em 57\% por quatro semanas antes da colheita, não obtiveram diminuição significativa no teor de nitrato nas folhas e nem na produção de matéria fresca da alface.

A substituição de $10 \%$ do $\mathrm{N}$ como amônio $\left(\mathrm{N}-\mathrm{NH}_{4}^{+}\right)$por todo o ciclo (T2) ou por 10 dias no final do ciclo (T3) foi efetiva na redução de nitrato na parte aérea da alface (Tabela 2). Houve redução significativa no teor de nitrato nas partes comestíveis, com $1920 \mathrm{mg} \mathrm{kg}^{-1}$ (T2) e $1756 \mathrm{mg} \mathrm{kg}^{-1}$ (T3). Da mesma forma o trabalho de Van der Boon et al. (1990) mostrou redução do teor de nitrato em folha de alface com o uso de nitrato associado com amônio em solução hidropônica. Fernandes et al. (2002) conseguiram redução do teor de nitrato em folhas das cultivares Regina e Grandes Lagos com a utilização de 190,4 mg $\mathrm{L}^{-1}$ de $\mathrm{N}$ em solução nutritiva, sendo 21,9\% como amônio em relação à utilização exclusiva de nitrato, com valor máximo de $1700 \mathrm{mg} \mathrm{kg}^{-1}$ de nitrato na matéria fresca. Os valores de nitrato em folhas obtidos por Fernandes et al. (2002) são similares aos tratamentos 2 e 3 deste experimento.

O consumo diário aceitável de nitrato preconizado pela Organização 
Mundial de Saúde, de 3,65 $\mathrm{mg} \mathrm{kg}^{-1}$ de massa corpórea por dia (Escoin-Peña $e t$ al. 1998), que equivale a $237,25 \mathrm{mg}$ para uma pessoa de $65 \mathrm{~kg}$. No caso deste experimento, uma pessoa com essa massa poderia consumir $78 \mathrm{~g}$ de alface por dia com $3024 \mathrm{mg} \mathrm{kg}^{-1}$ de nitrato (T4) ou $135 \mathrm{~g}$ da alface com $1756 \mathrm{mg} \mathrm{kg}^{-1}$ de nitrato nas folhas (T3).

Conclui-se que a utilização de $10 \%$ do $\mathrm{N}$ total da solução nutritiva como amônio, por todo o ciclo ou no final do ciclo, foi efetiva na redução do teor de nitrato nas folhas de alface, cv. Vera, sem prejuízo da matéria fresca. Por outro lado, a redução da concentração de nitrato na solução nos 10 dias do final do ciclo não reduziu o teor nitrato nas folhas.

\section{REFERÊNCIAS}

ANON J. 1997. The Commissions regulation (EC) Nr. 194/197. The Official of the European Community 31: 48-50.

BENNINI ERY; TAKAHASHI HW; NEVES CSVJ; FONSECA ICB. 2002. Teor de nitrato em alface cultivada em sistema hidropônico e convencional. Horticultura Brasileira 20: 183186.

BLOM-ZANDSTRA G; LAMPE JEM. 1985. The role of nitrate in the osmoegulation of lettuce (Lactuca sativa L.) grown at different light intensities. Journal of Experimental Botany 36: 1043-1052.
BLOM-ZANDSTRA M. 1989. Nitrate accumulation in vegetable its relationship to quality. Annals of Applied Biology 115: 553561.

CATALDO DA; HAROON M; SCHRADER LE; YOUNGS VL. 1975. Rapid colorimetric determination of nitrate in plant tissue by nitration of salicylic acid. Communications in Soil Science and Plant Analysis 6: 71-80.

CRADDOCK VM. 1983. Nitrosamines and human cancer: proof of an association. Nature 306: 638.

ECONOMAKIS CD; KOLEILAT R; CHARTZOULAKS KS. 1997. Effect of nitrogen concentration on growth, water, and nutrient uptake of lettuce plants in solution culture. Acta Horticulturae 449: 223-228.

ESCOIN-PEÑA MC; IBANEZ MAC; SANTAMARIA AA; LAZARO RC. 1998. Contenido de nitratos en lechugas y espinacas frescas. Alimentaria 29: 37-41.

FERNANDES AA; MARTINEZ HEP; PEREIRA, PRG; FONSECA MCM. 2002. Produtividade, acúmulo de nitrato e estado nutricional de cultivares de alface em hidroponia, em função de fontes de nutrientes. Horticultura Brasileira 2: 195-200.

FINE DH; ROSS D; ROUNBEHLER DP; SILVERGLEID A; SONG L. 1977. Formation in vivo of volatil $\mathrm{N}$-nitrosamines in man after ingestion of cooked bacon and spinach. Nature 265: 753-755.

FURLANI PR; SILVEIRA LCP; BOLONHEZI D; FAQUIM V. 1999. Cultivo hidropônico de plantas. Campinas: Instituto Agronômico. 52p.

JUNQUEIRA H. 1999. Hortaliças, novos caminhos no ambiente protegido. AGRIANUAL 99 - Anuário da Agricultura Brasileira. São Paulo: FNP Consultoria e Comercio. p.35-38.
KAFKAFI U. 1990. Root temperature, concentracion and ratio $\mathrm{NO}_{3} / \mathrm{NH}_{4}$ effect on plant development. Journal of Plant Nutrition 13: 1291-1305.

REININK K; EENINK AH. 1988. Genoptical difference in nitrate accumulation in shoots and roots of lettuce. Scientia Horticulturae 37: 1324.

LORENZ O. 1978. Potential nitrate levels in edible plants. In: D. Neilson \& J. MacDonald, Eds. Nitrogen in the Environment, Soil - Plant Nitrogen Relationship. New York: Academic Press. p.201-209.

McCALL D; WILLUMSEN J. 1998. Effects of nitrate and chloride application on the yield and nitrate content of soil-grown lettuce. Journal of Horticultural Science $e$ Biotechnology 73: 698-703.

SHENG MINGZHU. 1982. Study of the accumulation and content of nitrate in vegetable crops. Sica Horticulture 11: 257-262.

SHOUBECK MW; RIVERA R; BRIEN J; EBINGER S; DEGREGORIO RE. 1991. Variety selection and cultural methods for lowing nitrate levels in winter greenhouse lettuce and endive. Journal of Sustainable Agriculture 2: 49-75.

VAN DER BOON J; STEENHUIZEN JW; STEINGROVER EG. 1990. Growth and nitrate concentration of lettuce as affected by chloride concentration, $\mathrm{NH}_{4} / \mathrm{NO}_{3}$ rate and temperature of the recirculating nutrient solution. Journal of Horticultural Science 65: 309-321. 